\title{
Analysis of Influence Factors of Quality of Life of Lung Cancer Patients with Skin Adverse Drug Reactions Under Targeted Therapy
}

Ruofei Du ( $\sim$ ruofei2007666@163.com)

The college of nursing and health of Zhengzhou university

\section{Xin Wang}

The College of Nursing and Health of Zhengzhou University

Huiyue Zhou

The College of Nursing and Health of Zhengzhou University

\section{Lixia Ma}

School of Statistics, Henan University of Economics and Law

Leon M. Larcher

Centre for Comparative Genomics, Murdoch University

\section{Changying Chen}

The First Affiliated Hospital of Zhengzhou University

\section{Tao Wang}

The College of Nursing and Health of Zhengzhou University

\section{Research Article}

Keywords: NSCLC, Adverse drug reactions, Quality of Life, Targeted therapy, Influence factors.

Posted Date: January 3rd, 2022

DOI: https://doi.org/10.21203/rs.3.rs-1154197/v1

License: (c) (i) This work is licensed under a Creative Commons Attribution 4.0 International License. Read Full License 


\section{Abstract \\ Purpose}

This study was to assess the status of quality of life and explore the possible factors correlated with quality of life among non-small cell lung cancer (NSCLC) patients with skin adverse drug reactions under targeted therapy.

\section{Methods}

We performed a cross-sectional study including 536 NSCLC patients with skin adverse drug reactions by targeted therapy in cancer outpatient clinics of three hospitals in China between May 2020 and May 2021. And we collected data with structured questionnaires and identified the relationships among coping style, self-management and quality of life by Pearson correlation analysis and multiple linear regression algorithm.

\section{Results}

The total score of quality of life was $46 \pm 12.84$ in 536 NSCLC patients with skin adverse drug reactions undergoing targeted therapy. In multiple linear regression analysis, we identified the significant factors associated with quality of life including age, education level, combination of medicine, Charlson Comorbidity Index $(\mathrm{CCl})$, stages of disease, facing, yield, symptom management, daily activity management, psychological and emotional management, self-efficacy and self-management $(P<0.05)$.

\section{Conclusions}

NSCLC patients with skin adverse drug reactions undergoing targeted therapy generally had a compromised quality of life. And the critical factors that affected the status of quality of life were age, education level, co-morbidity, the combinatorial application of drugs and stage of disease, selfmanagement and coping styles.

\section{Introduction}

Lung cancer is the main cause of cancer-related death, accounting for a quarter of total cancer deaths. Globally, about 2.22 million new lung cancer cases and 1.55 million deaths from lung cancer were reported annually, with about 787.000 new lung cancer cases and 631.000 lung cancer-related deaths in China in 2015[1; 2]. Among various types of lung cancer, non-small cell lung cancer (NSCLC) accounts for $85 \%$ of the total cases, with the proportion of young patients ( $\leq 45 y)$ increasing gradually[3]. Importantly, $75 \%$ of NSCLC patients were in advanced stages when they were diagnosed with a 5 -year survival rate of about $15 \%[4]$. As a result, NSCLC-related treatment and clinical care have received great attention in 
recent years. Rapid advances in molecular biology over the past-half century have made it possible to identify molecular targets for cancer cells of particular types, thus providing a framework for targeted anticancer therapies[5][6]. Several studies have shown that targeted therapies could improve overall survival (OS), progression-free survival (PFS) and response rate (RR) of cancer patients, and improve patient tolerance and quality of life[7]. Epidermal growth factor receptor tyrosine kinase inhibitors (EGFRTKIs) are the most commonly used targeted therapy in the treatment of NSCLC. The National Comprehensive Cancer Network (NCCN) guidelines confirmed EGFR-TKIs as a standard first-line treatment for NSCLC patients with EGFR mutation[8], representing more than 50\% of NSCLC patients in the Asia-Pacific region[9]. Importantly, due to its oral administration route, EGFR-TKIs have become increasingly popular in recent years[10].

However, because EGFR signaling plays a critical role in epidermal physiology, the association of skin toxicity with EGFR inhibition is not an unexpected phenomenon. A recent investigation reported that about $80 \%$ of patients who received EGFR-TKIs treatment occurred skin adverse drug reactions (ADRs) within 3 months. The manifestations of skin ADRs associated with EGFR-TKIs include acneiform eruptions (even papule pustular rash), pruritus, dryness of skin, paronychia and nail loss, hair changes and hypersensitivity[11]. The severe skin ADRs hurt patients both physically and psychologically[12]. For example, the symptoms such as itchiness caused by severe skin ADRs can disturb sleep, and symptoms occurred on hands and feet negatively affected daily activities of patients[13]. Meanwhile, as skin condition plays an important role in body image and self-esteem, long-term and severe skin ADRs also affect the intimate and interpersonal relationships of patients[14], and result in increased psychological issues such as anxiety, and lead to social participation disorders[15]. Therefore, skin toxicities can have a profound impact on patients' quality of life (QoL) owing to symptoms caused and potential esthetic sequelae. Several studies indicated that among the patients receiving EGFR-TKIs-based targeted therapy, the medication adherence was unsatisfactory, about $32 \%$ of the patients stopped taking drugs due to poor QoL caused by various skin toxic reactions, which compromised the treatment effect and even leaded to cancer recurrence or progression[16]. As suggested by a randomized controlled trial, the level of QoL was positively correlated with RR and OS[17].

A couple of factors have been previously identified associated with the QoL of patients. As shown in a recent study, a positive coping style enabled patients to actively communicate disease with health providers and solve difficulties during symptom management[18]. In another study, it was reported that the negative effects of treatment and the distress induced by symptoms could be reduced through the adjustment of coping strategies. Indeed, a reasonable coping style might enable patients to better coordinate the relationship between the degree of ADRs and treatment and promote patients' Qo[19]. The self-management was another factor correlating with functional status in cancer patients with targeted therapies[20]. Self-management recommended by clinical guidelines including preventive measures and appropriate interventions may help to prevent and control severe skin reactions for improved QoL[21]. As demonstrated by Huang'sreport, good self-management could effectively regulate the troubles caused by ADRs of lung cancer treatment[22]. Additionally, a meta-analysis study indicated that patients with enough self-management ability showed less anxiety and better adaptability under stressful 
environments. And patients with effective self-management capacity tend to keep a positive state and could actively learn relevant knowledge and skills to improve QoL[23]. However, although factors such as coping style and self-management might significantly influence the QoL of patients with skin ADRs under targeted therapy, no comprehensive investigations in this area have been reported.

This study aimed to assess the level of QoL of NSCLC patients with skin ADRs undergoing targeted therapy and determine whether and how the coping style and self-management affect QoL. Then, based on the collected data, we endeavored to develop pertinent promotion strategies to reduce the severity of ADRs for improved QoL. The hypotheses were: (1) due to the negative effect of skin ADRs, NSCLC patients undergoing targeted therapy had low QoL; (2) patients with more positive coping styles tended to have better QoL; (3) patients with better self-management tended to have better QoL.

\section{Methods}

\section{Design And Setting}

This work employed a cross-sectional study from May 2020 to May 2021. Outpatient NSCLC patients with skin ADRs were recruited from three tertiary hospitals in Henan province to receive EGFR-TKIs treatment.

\section{Study Population}

A total of 536 NSCLC patients with skin ADRs undergoing EGFR-TKIs treatment were recruited from three hospitals in Henan, China. Convenience sampling was used. Inclusion criteria for participation in the study were: (1) the pathological diagnosis was NSCLC; (2) patients had with EGFR-TKIs treatment for at least 4 weeks; (3) patients had any skin ADR according to the common terminology criteria for adverse events version 5.0 (CTCAE 5.0); (4) patients were $18 \llbracket 80$ years old; (5) cognitive function was normal, and the patient could communicate normally; (6) informed consent of patients. Exclusion criteria were: (1) patients with poor physical function were difficult to complete the research; (2) patients were unable to complete the questionnaire independently or with the assistance of investigators.

The sample size was calculated based on $\mathrm{G}$ power 3.1.1 version with the effect size of 0.76 , a desired significance level of 0.05 and a power of 0.80 [24]. The estimated minimal sample size was 286. Among 700 NSCLC patients with EGFR-TKI treatment, 615 of them met the inclusion criteria and were contacted. As 43 of the contacted patients refused to participate and 36 did not complete all questionnaires, a total of 536 patients were included in this study.

\section{Procedure And Data Collection}


Totally 536 NSCLC patients were invited to participate in this study and informed the detailed study design. The potential participants were interviewed face to face by nurses when they attended the clinic. The principal investigator (PI) explained the purpose, content, and investigation procedures as well as the principle of anonymity of the study. And informed consent forms were signed by all participants. Questionnaires were distributed to the participants during their clinical consultation or after the clinical visit in the waiting room.

After obtaining the consent of relevant departments, we consulted the medical records of patients to get disease-related information. Trained postgraduate students were recruited to distribute questionnaires and give explanations to the patients' questions. For the illiterate participants, the researchers read the question items verbatim. And then responses were recorded on the questionnaire. The questionnaires were collected immediately after completion, checked for any missing information, and followed up with the participants.

\section{Measures}

\section{Demographic Characteristics}

This is a self-designed questionnaire to collect information of social demography variables, such as gender, age, level of education, family monthly income, marital status, occupation, residence, health insurance, disease stages, duration of treatment, combined drug application and comorbidities (calculated as the number of chronic diseases included in Charlson Comorbidity Index (CCI)).

\section{Functional Assessment of Cancer Therapy-Epidermal Growth Factor Receptor Inhibitor 18 (FACT-EGFRI-} 18)

The FACT-EGFRI-18 was used to investigate the QoL[25].The scale was divided into three dimensions: physiological status ( 7 items), social and family status ( 6 items) and functional status ( 5 items) to investigate the QoL of patients in the last 7 days. According to the degree of impact on patients' QoL, Likert 5-level scoring method was adopted ranging from "no impact" ( 0 point) to "a lot impact" (4 points). The forward items were scored $0 \llbracket 4$ points and the reverse items were scored $4 \llbracket 0$ points, the total score was obtained by summing the scores of each item. The total score range was $0 \otimes 72$. The higher the score, the better the QoL. The Cronbach's a coefficient of the total scale was 0.919.

\section{Self-management}

Self-management was evaluated by cancer patient Self-management Evaluation Scale developed by Cheng Lingling in 2017[26]. And the scale included 6 dimensions: daily life management (11 items), symptom management (7 items), emotional management (9 items), communication with medical staff (4 items), information management (3 items), and self-management efficacy (10 items). Likert 5-level 
scoring ranged from "no" (1 point) to "always" (5 points). The higher the score, the better the selfmanagement ability of patients. The Cronbach's a coefficient of the scale ranged from 0.698 to 0.933 , and the Cronbach's a coefficient of the total scale was 0.889 .

\section{Medical Coping Modes Questionnaire (Mcmq)}

MCMQ was used to measure the patients' coping styles[27]. It included 3 dimensions: face (8 items), avoid (7 items) and yield (5 items). A 4-point Likert scale was used for each item to measure agreement with each statement ( $1=$ never, $2=$ seldom, $3=$ sometimes, $4=$ always). The higher the cumulative score, the more the coping style. The Cronbach's a coefficients of the 3 dimensions were $0.84,0.83$ and 0.92 , respectively.

\section{Ethics Statement}

This study was approved by the Ethics Committee of the college of our university and administrative permissions were obtained from directors of oncology departments. All methods were carried out in accordance with relevant guidelines and regulations. Information about the study was provided to the participants and we got written informed consent from all participants prior to their inclusion in the study.

\section{Statistical Analysis}

All data were analyzed by the statistical software SPSS 13.0. Quantitative data was analyzed using descriptive statistical method to calculate sample demographics and clinical variables. In the descriptive analyses, means and standard deviations were calculated for continuous data while frequency and percentages for categorical variables. Pearson correlation analysis was applied to determine the correlation between patients' self-management and coping styles. And QoL.Multiple linear regression analysis was performed to explore factors independently related to QoL in patients with skin ADRs undergoing targeted therapy. All statistical tests used were two-tailed, and $P$-values $₫ 0.05$ were considered as statistical significance.

\section{Results}

\section{Demographical Characteristics}

In this study, a total of 572 questionnaires were distributed and of which 536 were completed with a response rate of $93.7 \%$. A total of 536 participants were enrolled in the study with more men $(53.2 \%)$ than women (46.8\%). The ages of these patients ranged from 41 to 79 years. And the mean age of the respondents was 63.6 years (SD $=8.32$ years), with $59.9 \%$ over 60 years old. Other key characteristics of the sample were also included as shown in Table 1. 
Table 1 Patient characteristics $(n=536)$

\begin{tabular}{|c|c|c|}
\hline Variables & & Frequency $₫ \% \rrbracket$ \\
\hline \multirow[t]{2}{*}{ Marital status } & With spouse & $450 \rrbracket 84.0 \rrbracket$ \\
\hline & Without spouse & $86 \rrbracket 16.0 \bigotimes$ \\
\hline \multirow[t]{3}{*}{ Education Level } & Junior school and below & $173 \rrbracket 32.3 \rrbracket$ \\
\hline & High school & $268 \rrbracket 50.0 \rrbracket$ \\
\hline & College and above & $95 \rrbracket 17.7 \rrbracket$ \\
\hline \multirow[t]{2}{*}{ Occupation status } & Employed & $34(6.3)$ \\
\hline & Unemployed & $502(93.7)$ \\
\hline \multirow[t]{2}{*}{ Residence } & Urban & 207凶38.6凶 \\
\hline & Rural & $329 \llbracket 61.4 \rrbracket$ \\
\hline \multirow[t]{2}{*}{ Medical insurance } & Yes & $417 \rrbracket 77.8 \rrbracket$ \\
\hline & No & $119 \rrbracket 22.2 \rrbracket$ \\
\hline \multirow[t]{4}{*}{ Family income Chinese RMB ( $¥) / M / P$} & $\otimes 1000 / \mathrm{M}$ & $27 \rrbracket 5.0 \rrbracket$ \\
\hline & $1001 \otimes 3000 / \mathrm{M}$ & 208囚38.8】 \\
\hline & $3001 \otimes 5000 / \mathrm{M}$ & $238 \rrbracket 44.4 \rrbracket$ \\
\hline & $\nabla 5001 / \mathrm{M}$ & $63 \rrbracket 11.8 \rrbracket$ \\
\hline \multirow[t]{2}{*}{$\mathrm{CCl}$} & $\otimes 2$ & $276 \rrbracket 51.5 \rrbracket$ \\
\hline & $\leq 2$ & $260 \rrbracket 48.5 \rrbracket$ \\
\hline \multirow[t]{2}{*}{ Duration of medicine (month,M) } & $\leq 3$ & $333 \rrbracket 62.1 \rrbracket$ \\
\hline & $₫ 3$ & $203 \llbracket 37.9 \rrbracket$ \\
\hline \multirow[t]{2}{*}{ Combination of medicine } & Yes & 297\55.4囚 \\
\hline & No & $239 \llbracket 44.6 \rrbracket$ \\
\hline \multirow[t]{4}{*}{ Stages of disease } & Stage $\otimes$ & 61ه11.4ه \\
\hline & 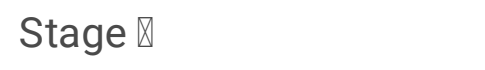 & $86 \rrbracket 16.0 \bigotimes$ \\
\hline & 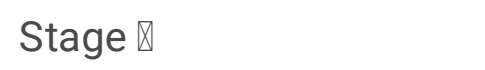 & $216 \varangle 40.3 \rrbracket$ \\
\hline & Stage $\otimes$ & 173囚32.3囚 \\
\hline
\end{tabular}


Quality of life of NSCLC patients with skin ADRs undergoing targeted therapy

The mean total score of QoL was 46 (12.84). And the lowest and highest scores were 27 and 70 , respectively. Details of QoL were demonstrated in Table 2.

Table 2

Quality of life of NSCLC patients with skin ADRs undergoing targeted therapy $(n=536)$

\begin{tabular}{|ll|}
\hline Items & Mean score $(\mathbb{\nabla x} \pm \mathbf{s})$ \\
\hline Physiological status & $18.29 \pm 4.95$ \\
\hline Social and family status & $14.37 \pm 4.67$ \\
\hline Functional status & $13.34 \pm 3.56$ \\
\hline Total score & $46 \pm 12.84$ \\
\hline
\end{tabular}

\section{Self-management And Coping Styles}

The mean value of the total score was $(129.31 \pm 30.71)$ for the patient's self-management, and the details of self-management and the coping styles were demonstrated in Table 3.

Table 3

Self-management and coping styles of NSCLC patients with skin ADRs undergoing targeted therapy $(n=536)$

\begin{tabular}{|ll|}
\hline Items & Mean score $(\mathbb{\nabla x \pm s )}$ \\
\hline Symptom management & $20.10 \pm 5.23$ \\
\hline Daily activity management & $34.86 \pm 8.70$ \\
\hline Psychological and emotional management & $25.43 \pm 7.64$ \\
\hline Communicate with medical staff & $11.55 \pm 3.55$ \\
\hline Information management & $9.19 \pm 2.83$ \\
\hline Self-efficacy & $28.18 \pm 7.81$ \\
\hline Total score of self-management & $129.31 \pm 30.71$ \\
\hline Facing & $18.29 \pm 4.95$ \\
\hline Avoidance & $14.37 \pm 4.67$ \\
\hline Yield & $13.34 \pm 3.56$ \\
\hline
\end{tabular}




\section{Correlation Analysis Among Self-management, Coping Styles And Qol}

Pearson's correlations among the main variables indicated that QoL was significantly positively correlated with symptom management $(r=0.746, P<0.01)$, daily activity management $(r=0.712, P<$ $0.01)$, psychological management $(r=0.614, P<0.01)$, emotional management $(r=0.667, P<0.01)$, information management $(r=0.685, P<0.01)$, self-efficacy $(r=0.643, P<0.01)$ and self-management $(r=0.785, p<0.01)$. However, there was no significant correlation between communicate with medical staff and QoL $(P>0.05)$. Facing was positively correlated with QoL $(r=0.807, P<0.01)$, but yield and avoidance were significantly negatively correlated with $Q \circ L(r=-0.718,-0.711, P<0.01)$.

\section{Factors Of Patients' Qol}

Multiple linear regression analysis was performed to examine QoL-related factors in NSCLC patients with skin ADRs undergoing targeted therapy. All variables including demographic characteristics, selfmanagement and coping strategies were entered by stepwise variable selection with the forward selection and backward elimination methods being combined to filter the independent variables. As shown in Table 4, the multiple linear regression analysis identified significant factors for QoL prediction. The model could explain $72 \%$ of the changes in QoL level. And the significant factors independently associated with QoL were as follows: age [(95\% Cl: 2.093-5.172); $P=0.000]$, education level [(95\% Cl: $1.089-2.226) ; P=0.034]$, combination of medicine [(95\% Cl: $1.070-3.880) ; P=0.001], \mathrm{CCl}[(95 \% \mathrm{Cl}$ : $1.145-2.039) ; P=0.031]$, disease of stages [(95\% Cl: $3.145-5.039) ; P=0.000]$, facing [(95\% Cl: $1.681-$ $6.185) ; P=0.000]$, yield [(95\% Cl: $-1.234-3.336) ; P=0.001]$, symptom management [(95\% Cl: $3.145-$ 6.039); $P=0.000$ ], daily activity management [(95\% Cl: $2.145-3.439) ; P=0.028]$, psychological and emotional management [(95\% Cl: -0.307--0.027); $P=0.020]$ and self-efficacy [ $(95 \% \mathrm{Cl}:-0.307--0.027) ; P$ $=0.020$ ]. Individuals with comorbidities, combination of medicine, older age, lower education level and worse disease stages were more likely to be worse QoL. Patients with better self-management and positive coping style had significantly higher possibility of high QoL. Table 5 showed the independent variables assignment in multiple linear regression analysis. 
Table 4

Multiple regression analysis of patients' QoL $(n=536)$

\begin{tabular}{|lllll|}
\hline Variables & B & SB & t & P \\
\hline Constant & 17.488 & 5.204 & 3.361 & 0.001 \\
\hline Age & 3.632 & 0.784 & 4.635 & 0.000 \\
\hline Education level & 1.157 & 0.544 & 2.128 & 0.034 \\
\hline CCI & 1.542 & 0.711 & 2.169 & 0.031 \\
\hline Stages of disease & 0.807 & 0.146 & 8.641 & 0.000 \\
\hline Combination of medicine & 2.475 & 0.715 & 3.461 & 0.001 \\
\hline Facing & 0.933 & 0.128 & 7.279 & 0.000 \\
\hline Yield & -0.785 & 0.229 & -3.433 & 0.001 \\
\hline Symptom management & 0.972 & 0.065 & 5.128 & 0.000 \\
\hline Daily activity management & 2.459 & 1.691 & 2.136 & 0.033 \\
\hline Psychological and emotional management & 0.167 & 0.071 & 2.399 & 0.020 \\
\hline Self-efficacy & 0.374 & 0.101 & 2.285 & 0.024 \\
\hline Self-management & 0.107 & 0.027 & 3.951 & 0.000 \\
\hline
\end{tabular}

Table 5 Independent variables assignment of multiple regression analysis 


\begin{tabular}{|c|c|}
\hline Independent Variables & Assignment \\
\hline Age & $\geq 60=1 ; \otimes 60=2$ \\
\hline Education level & $\begin{array}{l}\text { junior school or below=1; high school=2; college or } \\
\text { above }=3\end{array}$ \\
\hline $\mathrm{CCl}$ & $\nabla 2=1 ; \leq 2=2$ \\
\hline Combination of medicine & yes $=1 ;$ no $=2$ \\
\hline Stages of disease & Stage $\rrbracket=1 ;$ Stage $\rrbracket=2 ;$ Stage $\rrbracket=3 ;$ Stage $\rrbracket=4$ \\
\hline Facing & Continuous value \\
\hline Yield & Continuous value \\
\hline Symptom management & Continuous value \\
\hline Daily activity management & Continuous value \\
\hline $\begin{array}{l}\text { Psychological and emotional } \\
\text { management }\end{array}$ & Continuous value \\
\hline Self-efficacy & Continuous value \\
\hline Self-management & Continuous value \\
\hline
\end{tabular}

\section{Discussion}

NSCLC patients with targeted therapy usually have poor QoL due to skin ADRs. Identifying the factors correlated with QoL was critical for designing targeted management strategies. In the current crosssectional study, we explored self-management and coping styles as well as the association between them and QoL. And based on these results, the potential factors affecting the QoL were further investigated. As shown in our study, age, education level, co-morbidities, combination of medicine, stages of disease, facing, yield, symptom management, daily activity management, psychological/ emotional management and self-efficacy of self-management were crucial indicators in patients' QoL.

This study suggested a positive correlation between age and the QoL, with elderly patients showing poor QoL. One possible explanation is that older people were more likely to have concomitant diseases and their physical function deteriorated. At the same time, older people were more prone to age-related organ deterioration and poor tolerance, which may reduce QoL[28]. As confirmed by previous studies, patients with comorbidities and performing combined medicine applications normally had more complicated conditions, the interactions of diseases and drugs might lead to worse body function and aggravation of skin ADRs[29]. Our previous retrospective study indicated that the level of education affected the QoL, which was further confirmed in this study[30]. Indeed, poorly educated patients generally lacked the awareness to actively gain information to improve their QoL. As a result, they tended to find it difficult to 
accept and understand self-management. Additionally, late-stage diseases are correlated with more severe symptoms, and negatively impact patients not only physically but also psychologically, and lead to a decline in QoL.

The mean score of 46 (SD=12.84) for the QoL of the 536 participants as investigated in this study indicated that it was necessary to improve the QoL for NSCLC patients with skin ADRs undergoing targeted therapy. The symptoms of skin ADRs caused physical discomfort. The pruritus, irritation, sensitivity of skin often made patients upset which could disrupt the sleep cycle and daily activities[31]. Moreover, the xerosis, thinning, shriveling, darkening of skin can increase pressure in social life and lead to social participation barriers[32], these issues could be further enhanced by anxiety, depression, emotional instability and inferiority caused by the persistent helplessness to ADRs[33].

It should also be emphasized that QoL also affected patients' prognoses. As shown, poor QoL could make patients pessimistic about treatment and lead to treatment interruption [34]. In addition, poor QoL seriously affected the efficacy of anti-cancer therapy, and even led to cancer recurrence or progression[35]. Due to the limited studies worldwide evaluating the QoL of cancer patients undergoing targeted therapies, we were unable to comprehensively compare the QoL of patients in similar populations in different countries. However, in another study for lung cancer patients, the mean score of the QoL was 38 which was worse than reported in this study. One possible reason might be that the patients selected in that study were all advanced patients with poor physical function and nutritional status, which could affect the treatment tolerance of patients. Another reason might be that most patients of the study were combined with chemotherapy, and the side effects of chemotherapy could further reduce the QoL[36].

This study also demonstrated a relatively low self-management score among patients with targeted cancer therapy $(129.31, S D=30.71)$. As an important predictor of QoL in the clinical practice[37]. selfmanagement ability represents the knowledge, attitude and skills of patients in maintaining high QoL. According to a previously published study, patients with good self-management could effectively deal with adverse effects of lung cancer treatment and showed less anxiety and better adaptability under stressful environments[38]. Meanwhile, patients with good self-management paid more attention to their health status and adhered better to the advice given by medical staff. In this study, we found that the patient's management of symptoms, daily activities, self-efficacy, psychology and emotion could affect the QoL significantly. Patients who conducted good symptom management were aware of the signs of symptoms and were more likely to insist on assessing, monitoring and recording symptoms and adverse reactions. Once symptoms occurred, they could reduce the physical damage through pharmacological and non-pharmacological interventions[39].

For self-management, we'd better guide patients to strengthen the identification, monitoring, evaluation and recording of symptoms to prevent the aggravation of symptoms and adverse reactions. At the same time, health providers should train patients to develop medication management and adhere to good 
health behavior. In addition, the self-management of emotion, self-image and stress also should be considered.

Studies had shown that patients with high self-efficacy could monitor and manage their behavior well[40]. The self-efficacy reflected the patients' confidence in managing disease. Patients with better selfefficacy were always in a positive state to overcome difficulties and could actively learn relevant knowledge and skills to improve QoL. Negative psychological status might complicate the relationship between patients and caregivers, and had adverse effects on the anti-cancer treatment and disease management of patients. Patients immersed in bad emotions paid less attention to disease management, on the other side, bad emotions might affect the judgment of self-worth, resulting in the reduction of self-identity. All of these led to the decline of patients' QoL[41].

Adequate coping strategies are the premise of good QoL. In this study, we discovered that patients needed more positive coping strategies. Positive coping styles enhanced the ability and confidence of individuals to relieve pressure while negative coping styles strengthened negative psychology and reduced resilience. Patients with good coping skills such as facing were able to communicate and solve problems actively. Through the adjustment to positive coping styles, it could be reduced the negative effects of treatment and symptom distress[42]. This study showed that, when patients yield more, their QoL worse, on the contrary, when patients faced more, better their QoL. It had to do with Larsen, mobile health promotion strategies in the face of the problem of cancer patients with oral chemotherapy at home. In this way, patients could evaluate and cope with difficulties timely take measures correctly. Yield weakened patients' health responsibility, which might further make patients reluctant to do selfmanagement. During treatment, yield might reduce communication between the patients and others which hindered high quality and professional guidance. Moreover, if patients yielded, their consciousness of maintaining good QoL was worse, they might fail to self-examine which would cause recurrence of adverse events[43].

To our knowledge, this was the first study to describe the QoL of cancer patients with targeted therapy in China. The results of this study are enlightening: it is imperative to improve patients' self-management ability and evaluate the coping style of patients and help patients take positive coping styles which contribute to disease management. Furthermore, healthcare providers should develop personalized coping plans and guide patients on how to solve problems and overcome difficulties effectively. Additionally, we should take measures to promote communication between patients and others, and encourage patients to express their real thought to avoid yield. Moreover, providing social support, family support and peer support were important.

\section{Strengths And Limitations}

QoL is an important indicator to determine the patients' prognosis and therapeutic effect. Because of the high incidence of skin ADRs of cancer patients undergoing targeted treatment and the impact of skin ADRs on the QoL, it is necessary to know the level of QoL. This study can serve to evaluate the QoL of 
cancer patients with skin ADRs under targeted treatment. In addition, this study explored the factors that might affect the QoL of patients and identified some changeable factors, which laid the foundation for the development of targeted interventions. Besides, the sample size was large, and the credibility of the samples was strong.

However, there were also several limitations. Firstly, the sample was selected from three hospitals in one area without systematic sampling methods, which means that the possibility of selection bias. Secondly, the patients we chose only received one kind of targeted drug which might compromise the reproducibility of the results. Additionally, only two QoL affecting factors were systematically investigated, the relationship between these variables and QoL and the correlation and interrelationship among these variables should be explored in the future.

\section{Conclusion}

The level of QoL among NSCLC patients with skin ADRs undergoing targeted therapy is suboptimal and needs to be improved. Our findings indicated a significant association between age, education level, combination of drugs, comorbidities, stages of disease, coping styles, self-management and QoL in NSCLC patients undergoing targeted therapy. Our study provided preliminary information and recommendations for developing effective health care intervention programs.

\section{Declarations}

\section{Funding}

1. China Postdoctoral Science Foundation in 2018: (2018M630839)

2. The National Natural Science Foundation of China (No.81773175)

\section{Conflict of interest}

None declared.

\section{Availability of data and material}

The data generated during and/or analyzed during the current study are not publicly available, but are available from the corresponding author who was an organizer of the study.

\section{Code availability}

Not applicable.

\section{Authors' contributions}

All authors have read and approved the manuscript.WT and CCY were responsible for the overall design and quality control of the study as well as the communication with the hospital and departments 
reviewed.DRF, WX and WT made substantial contributions to conception and design, or acquisition of data, or analysis and interpretation of data; WX, LML and MLX involved in drafting the manuscript or revising it critically for important intellectual content; $Z \mathrm{ZHY}$ contributed to the training and management of nurses and students who collected data. DRF, ZHY, WX, LML, CCY and WT had given final approval of the version to be published. MLX contributed to data analysis and solving statistic problems. Each author should have participated sufficiently in the work to take public responsibility for appropriate portions of the content; DRF, CCY and WT agreed to be accountable for all aspects of the work in ensuring that questions related to the accuracy or integrity of any part of the work are appropriately investigated and resolved.

\section{Ethics approval}

This study was approved by the Ethics Committeeof the college of our universityand administrative permissions were obtained from directors of oncology departments. All methods were carried out in accordance with relevant guidelines and regulations. Information about the study was provided to the participants and we got written informed consent from all participants prior to their inclusion in the study.

\section{Consent to participate}

Not applicable.

\section{Consent for publication}

Not applicable.

\section{Acknowledgement}

We thank the First Affiliated Hospital of Zhengzhou University and Henan tumor hospital and the First Affiliated Hospital of Henan University of science and technology for their assistance and support, and the Follow-up Registration System and data provided by the Follow-up Center of the First Affiliated Hospital of Zhengzhou University. We also thank the oncologists and nurses of the three hospitals. We appreciated all participants for their generous participation.

\section{References}

1. J Kastner, R Hossain, C Whilte (2019) Semin. Roentgenol. 1(55): 36-63.

2. D Yang, Y Liu, C Baia, X Wang (2020) Cancer Lett. 468(2): 82-87.

3. M Galvez-Nino, R Ruiz, JA Pinto, K Roque, L Mas (2020) Lung Cancer 198(1): 195-200.

4. A Thomas, Y Chen, T Yu, M Jakopovic (2015) Front in oncol 5(33): 113-119.

5. X Liu, X Luo, C Jiang, H Zhao (2019) Clin. Genet. 95(6): 569-574.

6. GM Wilkes (2018) Asia-Pacific Journal of Oncology Nursing 5(2): 137-155. 
7. G Singal, PG Miller, V Agarwala, G Li, G Kaushik, D Backenroth, A Gossai, GM Frampton, AZ Torres, EM Lehnert (2019) JAMA The Journal of the American Medical Association 321(14): 1391-1402.

8. DS Ettinger, DE Wood, W Akerley, LA Bazhenova (2016) Journal of the National Comprehensive Cancer Network Jnccn 14(3): 255-264.

9. MC Wang, CL Wang, TL Chen, WC Chang, JJ Lu, PY Chang, CC Chiou (2017) Clinical Chemistry \& Laboratory Medicine 55(12): 1979-1986.

10. HP Kale, AP Mays, PA Nadpara, PW Slattum (2019) Urologic Oncology: Seminars and Original Investigations 356(37): 19-28.

11. Y Peng, Q Li, J Zhang, W Shen, X Zhang, C Sun, H Cui (2018) Biosci. Trends 33(32): 3817-3825.

12. Jung, Lim, Lee, Younhee, Jeong (2018) Cancer Nurs..

13. DM Barrios, GS Phillips, A Freites-Martinez, M Hsu, K Ciccolini, LA Skripnik, MA Marchetti, AM Rossi, EH Lee, L Deng, A Markova, PL Myskowski, ME Lacouture (2020) J Eur Acad Dermatol Venereol 34(6): 1340-1347.

14. K Yagasaki, H Komatsu, K Soejima, K Naoki, Y Hamamoto (2018) Asia-Pacific Journal of Oncology Nursing 5(2): 172-177.

15. J CHAN, Y LEE, C LIU, H SHIH (2019) J. Nurs. Res. 27(6): 51-59.

16. K Sano, K Nakadate, K Hanada (2020) BMC Cancer 20(1): 279-287.

17. H Staats, C Cassidy, J Kelso, S Mack, J Nemunaitis (2020) Biomedicine 5(13): 27-49.

18. Liao, Yu-Chien, Wei-Yu, Sun, Jia-Ling, Ko, Jen-Chung, Yu, Chong-Jen (2018) Supportive Care in Cancer 26(2): 989-996.

19. M Yuan, LL Huang, JH Chen, J Wu, Q Xu (2019) Signal Transduction and Targeted Therapy 55(5): 103-111.

20. Ilufredo Y Tantoy, Janine K Cataldo, Bradley E. Aouizerat, Anand Dhruva (2016) Cancer Nurs. 39(6): 437-445.

21. ME Lacouture, M Anadkat, A Jatoi, T Garawin, C Bohac, E Mitchell (2018) Clin. Colorectal Canc. 17(2): 85-96.

22. FF Huang, Q Yang, J Zhang, XY Han, JP Zhang, A Chirico (2018) PLoS One 13(9): 332-339.

23. A Chirico, F Lucidi, T Merluzzi, F Alivernini, A Giordano (2017) Oncotarget 8(22): 36800-36811.

24. F Faul, EED Lder, A Buchner, AG Lang (2009) Behav Res Methods 41(4): 1149-1160.

25. LI Wagner, SR Berg, M Gandhi, FJ Hlubocky (2013) Support. Care Cancer 21(4): 1033-1041.

26. C Lingling, S Yuqian. (2017) Chinese Journal of nursing 52(9): 1082-1087.

27. Shen Xiaohong, Jiang Qianjin (2000) Chinese Journal of behavioral medicine and brain science 9(1): 18-20.

28. TA Barnes, E Amir, AJ Templeton, S Gomez-Garcia, B Navarro, B Seruga, A Ocana (2017) Cancer Treat. Rev. 56(6): 1-7.

29. K Yamamoto, I Yano (2018) Med. Oncol. 35(2): 16-26. 
30. R Du, X Wang, L Ma, LM Larcher, T Wang (2021) BMC Cancer 21(1): 231-239.

31. DM Barrios, GS Phillips, A Freites-Martinez, M Hsu, ME Lacouture (2019) J. Eur. Acad. Dermatol. 34(6): 27-39.

32. JC Chan, YH Lee, CY Liu, HH Shih, WR Tang (2019) J. Nurs. Res. 27(6): 51-59.

33. K Yagasaki, H Komatsu, K Soejima, K Naoki, Y Hamamoto (2018) Asia-Pacific Journal of Oncology Nursing 5(2): 172-177.

34. H Komatsu, K Yagasaki, T Yamaguchi, A Mori, K Tamura (2020) Eur. J. Oncol. Nurs. 23(8): 10171028.

35. Jung, Lim, Lee, Younhee, Jeong (2018) Cancer Nurs. 42(6): 475-483.

36. Y He, H Jian, M Yan, J Zhu, J Chen (2019) BMJ Open 9(5): 236-242.

37. A Deutsch, NR Leboeuf, ME Lacouture, BN Mclellan (2020) American Society of Clinical Oncology Educational Book 40(40): 485-500.

38. V Sun, A Reb, M Debay, M Fakih, B Ferrell (2021) J. Cancer Educ. 25(4): 788-796.

39. Chen, Qiu, Di, Shao, Ying, Yao, Yue, Zhao, Xiaoying, Zang (2019) Quality of Life Research An International Journal of Quality of Life Aspects of Treatment Care \& Rehabilitation 67(5): 1245-1253.

40. FF Huang, Q Yang, J Zhang, XY Han, JP Zhang, M Ye (2018) Psychol. Health Med. 6(25): 11-18.

41. AC Lopes, R Bacalhau, M Santos, M Pereira, MG Pereira (2020) Journal of Clinical Psychology in Medical Settings 27(3): 603-614.

42. Liao, Yu-Chien, Wei-Yu, Sun, Jia-Ling, Ko, Jen-Chung, Yu, Chong-Jen (2018) Supportive Care in Cancer 12(23): 236-243.

43. W Huang, J Li, F Qiu, X Wu, S Zhu (2020) J. Clin. Pharm. Ther. 45(12): 29-39. 